\title{
A Note on Perturbations During a Regular Bounce
}

\author{
Thorsten J. Battefeld 1)* and Ghazal Geshnizjani ${ }^{2) \dagger}$ \\ 1) Physics Department, Brown University, Providence RI 02912 USA, and \\ 2) Department of Physics, University of Wisconsin, Madison WI 53706 USA.
}

(Dated: July 7, 2018)

\begin{abstract}
We point out an inconsistency in a method used in the literature for studying adiabatic scalar perturbations in a regular bouncing universe (in four dimensions). The method under scrutiny consists of splitting the Bardeen potential into two pieces with independent evolutions, in order to avoid a singular behavior at the boundaries of the region where the null energy condition (NEC) is violated. However, we argue that this method violates energy-momentum conservation.

We then introduce a novel method which provides two independent solutions for the Bardeen potential around the boundaries, even in the case of adiabatic perturbations. The two solutions are well behaved and not divergent.
\end{abstract}

PACS numbers: 04.50.+h,98.80.-k,11.25.Wx,98.80.Es

In recent years, regular toy-models of a bouncing universe have received a lot of attention due to string inspired singular bouncing models such as the cyclic scenario. Of particular interest is the evolution of scalar perturbations, since their spectrum is directly observable in the anisotropies of the cosmic background radiation and the large scale structure of the universe. The majority of toy models are four dimensional, requiring at least two matter fields, of which at least one has to violate the null energy condition (NEC).

If one focuses on adiabatic perturbations, the evolution equation for the Bardeen potential becomes singular at the boundaries of the NEC violating region before and after the bounce. One way out of this dilemma was advocated in [1] and subsequently used e.g. in [2, 3, 4]: One splits the Bardeen potential in two components, each of which satisfies a regular second order differential equation.

In this note we first show that this method is inconsistent because the fluid conservation equations are violated. Therefore all models using this method have to be re-evaluated. We then introduce a novel method, providing two independent and well behaved solutions for the Bardeen potential around the boundaries of the region with NEC violation, even in the case of adiabatic perturbations.

For simplicity, we work with a two fluid model with $T_{\nu}^{\mu}=T_{(a) \nu}^{\mu} \pm T_{(b) \nu}^{\mu}$ and equations of state $p_{(l)}=w_{l} \rho_{(l)}$ for $l=a, b$. Note that a bounce for the scale factor $a$ occurs only in case of a negative sign in front of $T_{(b) \nu}^{\mu}[\underline{8}]$. Perturbing the metric

$$
d s^{2}=a^{2}\left[(1+2 \Phi) d \eta^{2}-(1-2 \Phi) \delta_{i j} d x^{i} d x^{j}\right]
$$

where $\Phi$ is the Bardeen potential (longitudinal gauge, no anisotropic stress, see [ 6] for details), and perturbing also

\footnotetext{
*battefeld@physics.brown.edu

†ghazal@physics.wisc.edu
}

the energy momentum tensors

$$
\left(\delta T_{(l) \nu}^{\mu}\right)=\rho_{(l)}\left(\begin{array}{cc}
-\xi_{(l)} & \left(1+w_{l}\right) V_{(l), i} \\
-\left(1+w_{l}\right) V_{(l), i} & \left(w_{l} \xi_{(l)}\right) \delta_{j}^{i}
\end{array}\right)
$$

where $\delta p_{(l)}=w_{l} \delta \rho_{(l)}$ and $\xi_{(l)}:=\delta \rho_{(l)} / \rho_{(l)}$, the perturbed Einstein equations for $\mathcal{K}=0$ (a spatially flat universe) read

$$
\begin{gathered}
\nabla^{2} \Phi-3 \mathcal{H}\left(\mathcal{H} \Phi+\Phi^{\prime}\right)=\frac{a^{2}}{2} \kappa^{2}\left(\rho_{(a)} \xi_{(a)} \pm \rho_{(b)} \xi_{(b)}\right) \\
\begin{aligned}
\Phi^{\prime \prime}+3 \mathcal{H} \Phi^{\prime}+ & \left(2 \mathcal{H}^{\prime}+\mathcal{H}^{2}\right) \Phi=\frac{a^{2}}{2} \kappa^{2}\left(w_{a} \xi_{(a)} \rho_{(a)}\right. \\
& \left. \pm w_{b} \xi_{(b)} \rho_{(b)}\right)
\end{aligned} \\
{\left[\Phi \mathcal{H}+\Phi^{\prime}\right]_{, i}=-\frac{a^{2}}{2} \kappa^{2}\left(\rho_{(a)} V_{(a), i}\left(1+w_{a}\right)\right.} \\
\left. \pm \rho_{(b)} V_{(b), i}\left(1+w_{b}\right)\right)
\end{gathered}
$$

with $\kappa^{2}=8 \pi / M_{p}^{2}$. The energy conservation equations for each fluid, assuming no non-gravitational interactions between the fluids, are

$$
\rho_{(l)}\left(\left(1+w_{l}\right)\left[\nabla^{2} V_{(l)}-3 \Phi^{\prime}\right]+\xi_{(l)}^{\prime}\right)=0 .
$$

In the long wavelength limit the above relation yields

$$
\frac{\xi_{(l)}^{\prime}}{1+w_{l}}=3 \Phi^{\prime}
$$

which is another way of stating entropy conservation, that is

$$
S^{\prime}=\frac{\xi_{(a)}^{\prime}}{1+w_{a}}-\frac{\xi_{(b)}^{\prime}}{1+w_{b}}=0 .
$$

One can simply impose the adiabaticity condition by setting initially $S\left(\eta_{i n}\right):=0$ and thus $[9]$

$$
\frac{\xi_{(a)}}{1+w_{a}}=\frac{\xi_{(b)}}{1+w_{b}}
$$


has to hold.

Substituting this result back into (31) and (44) and then combining the two, one arrives at a second order equation for $\Phi$ :

$$
\begin{aligned}
& 0=\Phi^{\prime \prime}\left[\rho_{a}\left(1+w_{a}\right) \pm \rho_{b}\left(1+w_{b}\right)\right] \\
& +3 \mathcal{H} \Phi^{\prime}\left[\rho_{a}\left(1+w_{a}\right)^{2} \pm \rho_{b}\left(1+w_{b}\right)^{2}\right] \\
& +\left[-\left(w_{a}\left(w_{a}+1\right) \rho_{a} \pm w_{b}\left(w_{b}+1\right) \rho_{b}\right) \nabla^{2}\right. \\
& +2 \mathcal{H}^{\prime}\left(\rho_{a}\left(1+w_{a}\right) \pm \rho_{b}\left(1+w_{b}\right)\right) \\
& \left.+\mathcal{H}^{2}\left(\rho_{a}\left(1+w_{a}\right)\left(1+3 w_{a}\right) \pm \rho_{b}\left(1+w_{b}\right)\left(1+3 w_{b}\right)\right)\right] \Phi .
\end{aligned}
$$

In case of a minus sign, which is needed for a bounce to occur, or some negative $w_{i}$ this equation becomes singular at the boundaries of the NEC violating region (see also $\underline{3}$ ).

In the appendix of [1] a split of $\Phi$ into $\Phi_{a}+\Phi_{b}$ is suggested, such that each $\Phi_{l}$ satisfies

$$
\begin{aligned}
& \nabla^{2} \Phi_{l}-3 \mathcal{H}\left(\mathcal{H} \Phi_{l}+\Phi_{l}^{\prime}\right)=\frac{a^{2}}{2} \kappa^{2} \rho_{(l)} \xi_{(l)}, \\
& \Phi_{l}^{\prime \prime}+3 \mathcal{H} \Phi_{l}^{\prime}+\left(2 \mathcal{H}^{\prime}+\mathcal{H}^{2}\right) \Phi_{l}=\frac{a^{2}}{2} \kappa^{2} w_{l} \xi_{(l)} \rho_{(l)} .
\end{aligned}
$$

By adding these equations one arrives at the original Einstein equations (31) and (4). Combining (11) and (12) one can derive for each $\Phi_{l}$ the equation

$$
\begin{aligned}
0= & \Phi_{l}^{\prime \prime}+3 \mathcal{H}\left(1+w_{l}\right) \Phi_{l}^{\prime} \\
& +\left(-w_{l} \nabla^{2}+2 \mathcal{H}^{\prime}+\left(1+3 w_{l}\right) \mathcal{H}^{2}\right) \Phi_{l} .
\end{aligned}
$$

Note that these equations are regular for each $\Phi_{l}$. This is already the first hint that the method is doubtful, since the singular behavior vanished miraculously. Another reason to doubt this method is the fact that by just using equations (3) and (4), with no extra constraint like conservation of energy for each fluid or adiabaticity, one seems to be able to calculate the evolution of each $\Phi_{l}$ and subsequently $\Phi$ itself. However, it is obvious that in the equations (3) and (4) three unknowns appear, so that two equations are insufficient to calculate their evolution. Therefore one can not expect the solutions of (11) and (12) to be consistent with the conservation of the energymomentum tensor or other constraint equations.

One easy way to see this inconsistency is to look at the solutions in the long wavelength limit where equation (111) simplifies to

$$
-3 \mathcal{H}\left(\mathcal{H} \Phi_{l}+\Phi_{l}^{\prime}\right)=\frac{a^{2}}{2} \kappa^{2} \rho_{(l)} \xi_{(l)} .
$$

Adding the time derivative of the above equation to $\mathcal{H} \times$ itself and $3 \mathcal{H} \times(12$ yields

$$
\begin{gathered}
\rho_{(a)} \xi_{(a)}^{\prime}=3 \Phi_{a}^{\prime}\left(\rho_{(a)}\left(1+w_{a}\right)+\rho_{(b)}\left(1+w_{b}\right)\right), \\
\rho_{(b)} \xi_{(b)}^{\prime}=3 \Phi_{b}^{\prime}\left(\rho_{(b)}\left(1+w_{b}\right)+\rho_{(a)}\left(1+w_{a}\right)\right),
\end{gathered}
$$

where we also used the background Einstein equations and $\rho_{i}^{\prime}=-3 \mathcal{H}\left(1+w_{i}\right) \rho_{i}$. This result, together with the conservation equation (7), implies that the solutions of (13) have to satisfy

$$
\frac{\Phi_{a}^{\prime}}{\rho_{(a)}\left(1+w_{a}\right)}=\frac{\Phi_{b}^{\prime}}{\rho_{(b)}\left(1+w_{b}\right)} .
$$

One might think that this constraint is satisfied in the case of adiabatic perturbations, but it is not as we shall see now. Using (9) in (14) yields

$$
\frac{\mathcal{H} \Phi_{a}+\Phi_{a}^{\prime}}{\rho_{(a)}\left(1+w_{a}\right)}=\frac{\mathcal{H} \Phi_{b}+\Phi_{b}^{\prime}}{\rho_{(b)}\left(1+w_{b}\right)}
$$

which can further be simplified by using (17) to

$$
\frac{\Phi_{a}}{\rho_{(a)}\left(1+w_{a}\right)}=\frac{\Phi_{b}}{\rho_{(b)}\left(1+w_{b}\right)} .
$$

This together with (17) implies

$$
\frac{\Phi_{a}^{\prime}}{\Phi_{a}}=\frac{\Phi_{b}^{\prime}}{\Phi_{b}}
$$

yielding $\Phi_{a} \propto \Phi_{b}$. However, this is clearly in contradiction to (19), since the densities have a different dependency on conformal time if $w_{a} \neq w_{b}$. Therefore the splitting method itself is inconsistent and can not be trusted to regularize (10).

We propose another mathematical technique that can be used instead to approximate the solutions of (10) in the vicinity of $\rho_{t o t}+p_{t o t}=0$ at $\eta_{n e c}$. Equation (10) is a second order differential equation that in fourier space (suppressing the subscript on $\Phi_{k}$ ), has the following general form:

$$
A(\eta) \Phi^{\prime \prime}+B(\eta) \Phi^{\prime}+C(k, \eta) \Phi=0,
$$

where $A$ and $B$ are related via

$$
B=-A^{\prime},
$$

since energy conservation for each fluid requires [10]

$$
\rho_{l}^{\prime}=-3 \mathcal{H} \rho_{l}\left(1+w_{l}\right) .
$$

Our first goal is to derive one of the solutions around $\eta_{\text {nec }}$ perturbatively. We can then obtain the second solution by means of the Wronskian method.

Since $a\left(\eta_{\text {nec }}\right) \neq 0$ we can Taylor expand $\rho_{l}$ and consequently $A, B$ and $C$ around $\eta_{\text {nec }}$ :

$$
\begin{aligned}
& A(\delta)=A_{1} \delta+A_{2} \delta^{2}+A_{3} \delta^{3}+\ldots, \\
& B(\delta)=-A_{1}-2 A_{2} \delta-3 A_{3} \delta^{2}+\ldots, \\
& C(\delta)=C_{0}+C_{1} \delta+C_{2} \delta^{2}+\ldots,
\end{aligned}
$$

where we defined $\delta=\eta-\eta_{\text {nec }}$ and used $A\left(\eta_{\text {nec }}\right)=0$. We assume that $A_{1}$, corresponding to the linear term of $A(\delta)$, does not vanish. This is usually the case and plays a crucial role in our analysis. 
Furthermore, we assume the existence of an analytic solution around $\eta_{\text {nec }}$ (we will see bellow that it is a justified assumption [11]), so that it can be written as

$$
\Phi_{1}=\alpha_{0}+\alpha_{1} \delta+\alpha_{2} \delta^{2}+\ldots .
$$

Substituting relations (24)-(27) into (21) we obtain the following relations from the zeroth and first order equations in $\delta$

$$
\begin{aligned}
& \mathcal{O}\left(\delta^{0}\right) \quad \Rightarrow \quad 0=-A_{1} \alpha_{1}+C_{0} \alpha_{0} \\
& \mathcal{O}\left(\delta^{1}\right) \quad \Rightarrow \quad 0=\left(-2 A_{2}+C_{0}\right) \alpha_{1}+C_{1} \alpha_{0} .
\end{aligned}
$$

In general, this can only be satisfied if $\alpha_{0}=\alpha_{1}=0$. Fortunately, this does not imply $\Phi_{1} \equiv 0$, because the equations of higher order in $\delta$ can all be satisfied recursively. In fact, we can compute a complete power series solution for $\Phi_{1}$

$$
\begin{aligned}
\mathcal{O}\left(\delta^{2}\right) & \Rightarrow \alpha_{3}=\frac{2 A_{2}-C_{0}}{3 A_{1}} \alpha_{2} \\
& \vdots \\
\mathcal{O}\left(\delta^{n}\right) & \Rightarrow \alpha_{n+1}=\frac{\sum_{i=2}^{n}\left[i(3+n-2 i) A_{n+2-i}-C_{n-i}\right] \alpha_{i}}{(n-1)(n+1) A_{1}}
\end{aligned}
$$

with the consequence

$$
\Phi_{1}=\delta^{2}+\frac{2 A_{2}-C_{0}}{3 A_{1}} \delta^{3}+\ldots .
$$

Knowing one of the solutions of (21), $\Phi_{1}$, we can easily obtain the other solution, $\Phi_{2}$, by using the Wronskian technique. The Wronskian for a second order differential equations is defined as

$$
W=\Phi_{2}^{\prime} \Phi_{1}-\Phi_{1}^{\prime} \Phi_{2},
$$

where $\Phi_{1}$ and $\Phi_{2}$ are the independent solutions of (21). Henceforth, one can calculate $\Phi_{2}$ in terms of $W$ and $\Phi_{1}$ :

$$
\Phi_{2} \sim \Phi_{1} \int \frac{W}{\Phi_{1}^{2}} d \eta
$$

$W$ itself satisfies the first order differential equation

$$
A(\eta) W^{\prime}+B(\eta) W=0 .
$$

By substituting $B(\eta)$ from (22) we can solve the above equation for $W$ to

$$
W(\eta)=\beta A(\eta),
$$

where $\beta$ is just a constant. Combining this result for $W(\eta)$ with (33) and our solution for $\Phi_{1}$ from (31), we end up with

$$
\Phi_{2}=-\frac{A_{1}}{2}-\frac{8 A_{2}-C_{0}}{6} \delta+A_{3} \delta^{2} \ln (|\delta|)+O\left(\delta^{2}\right) .
$$

Note that although this solution is not analytic at $\eta=$ $\eta_{\text {nec }}(\delta=0)$, it is well behaved in the sense that both, the solution and its first derivative, remain continuous and finite. Thus, we have constructed the approximate form of the two independent solutions of (21) or subsequently (10). These can be used to match the solution on different sides of $\eta_{n e c}$.

To summarize, we have shown explicitly that the splitting method, first introduced in [1] and used to show the regularity of the Bardeen potential, is intrinsically inconsistent. All models using this method have to be re-evaluated [12], e.g. by using the technique introduced in this draft or by including entropy perturbations, as emphasized in [4] and later on in [5] (they derived a regular forth order equation for the full Bardeen potential).

\section{Acknowledgments}

We would like to thank the referee of [2] for drawing our attention to a possible problem associated with the splitting method, and N. Afshordi, D. Battefeld, R. Brandenberger and D. Chung for comments on the draft.
[1] P. Peter and N. Pinto-Neto, Phys. Rev. D 66, 063509 (2002) arXiv:hep-th/0203013.

[2] T. J. Battefeld and G. Geshnizjani,

[3] F. Finelli, JCAP 0310, 011 arXiv:hep-th/0307068.

[4] Patrick Peter, Nelson Pinto-Neto, Diego A. Gonzalez, JCAP 0312:003,2003. hep-th 0306005

[5] N. Pinto Neto, Int. J. Mod. Phys. D 13, 1419 (2004) arXiv:hep-th/0410225.

[6] V. F. Mukhanov, H. A. Feldman and R. H. Brandenberger, Phys. Rept. 215, 203 (1992).

[7] V. Bozza and G. Veneziano, arXiv:gr-qc/0506040

[8] $w_{b}=2 w_{a}+1$ corresponds to the bounce in [2] and $w_{b}=$ $2 w_{a}+1 / 3$ corresponds to the one in 3 .
[9] While one can not use the energy conservation equations for separate fluids in the case discussed in [2] to derive this condition, one can still arrive at the same relation due to the specific form of the corrections to the energy momentum tensor in 2].

[10] Again, although the energy conservation argument does not hold for 2] this relation still remains valid.

[11] Arguments in favor of an analytic $\Phi$ where given in e.g. [4] or 7], without relying on the splitting method.

[12] The model in [2] V.2 by the authors of this critique will be corrected shortly in the upcoming revision V.3. The background solution in 2], as well as the sections on vector and tensor perturbations are unaffected by this revision. 